\title{
Measurement of surface topography by RGB Shadow-Moiré with direct phase demodulation
}

\author{
José A. Gómez-Pedrero,*, Juan A. Quiroga ${ }^{\mathrm{a}}$, \\ M. José Terrón-López ${ }^{\mathrm{b}}$, Daniel Crespo ${ }^{\mathrm{a}}$ \\ ${ }^{a}$ Optics Department, Facultad de CC. Fisicas, Universidad Complutense de Madrid, \\ Ciudad Universitaria s/n, 28040 Madrid, Spain \\ ${ }^{\mathrm{b}}$ Electronic and Communications Department, Escuela Superior Politécnica, \\ Universidad Europea de Madrid, 28670-Villaviciosa de Odón, Spain
}

Received 16 September 2005; accepted 22 December 2005

Available online 9 March 2006

\begin{abstract}
In this paper we present the application of a direct demodulation method for the measurement of surface topography by means of Shadow-Moiré. In our set-up, we use three LEDs (with green, red and blue peak wavelengths) to illuminate the grating. Due to the different position of these light sources, a polychromatic Shadow-Moiré fringe pattern is produced, which can be described as the superposition of three monochromatic (red, green and blue) fringe patterns. Taking the image of this polychromatic fringe pattern with a RGB CCD camera, we get a monochromatic fringe pattern stored at each RGB channel of the $\mathrm{CCD}$. The direct demodulation algorithm employed uses these fringe patterns to calculate the wrapped phase map. After unwrapping the phase map using a standard multi-grid technique, we implemented an automatic procedure to detect the area of interest of the phase map by removing low modulation zones and to calculate the absolute value of the phase. In this way it is possible to determine the topography of a surface with a single RGB snapshot maintaining a simple experimental set-up, which is an important feature, especially for the study of dynamic
\end{abstract}

\footnotetext{
*Corresponding author. Fax: + 3413944674.

E-mail address: jagomezp@fis.ucm.es (J.A. Gómez-Pedrero).
} 
phenomena such as deformations. We present the experimental results obtained after measuring different objects with both smooth and rough surface textures.

(C) 2006 Elsevier Ltd. All rights reserved.

Keywords: Shadow-Moiré; RGB illumination; Asynchronous phase demodulation

\section{Introduction}

Shadow-Moiré is a well-known optical technique [1] for measuring the shape of a surface. It makes use of the Moiré pattern phenomena, which results from the superposition of a Ronchi grating and its shadow projected on the surface under test. The advantage of Shadow-Moiré against other alternative optical techniques, such as interferometry or holography, is the use of a simple experimental set-up with no special requirements for light coherence, mechanical isolation, etc. Therefore, Shadow-Moiré is well adapted for an industrial environment and constitutes a widely employed technique for optical inspection in fields like automotive manufacturing, aerospace industry, etc.

Conversely, one disadvantage of Shadow-Moire is the extraction of quantitative information from the fringe pattern produced by the Ronchi grating and its shadow. The difficulty arises from the special relationship between the phase of the ShadowMoire fringe pattern and the parameters of the experiment: master grid spacing and relative position of the master grid, test surface, light source and camera. Due to this, phase extraction techniques commonly employed in other experimental set-ups such as phase sampling, are difficult to apply to Shadow-Moiré.

Let us examine first the phase-shifting techniques. It is well known that these techniques require the controlled variation of at least one of the experimental parameters, introducing in this way a global phase shift equal for every point of the fringe pattern. It is necessary to know accurately the amount of the phase shift introduced in order to extract the phase properly. For example, in a Michelson interferometer, it is possible to introduce a global phase shift by displacing one of the mirrors located at the end of the interferometer arms [2-4], by means of PZT displacement device. In such a way, phase-shifts ranging from 0 to $2 \pi$ radians can be introduced with good accuracy [2-4].

To apply phase-shifting technique to Shadow-Moiré experiments it is necessary to introduce a global phase variation [5-8]. Two requirements should be met: (1) phase variation should be the same for every point of the fringe pattern and (2) the phaseshift introduced should be proportional to the variation of the experimental parameter. As it is stated in [5], these requirements can only be fulfilled under certain approximations thus a systematic error source is introduced in the measurement process [5-7]. Other possibility is described in [8] and consists in the simultaneous variation of two parameters: the light source height, and the distance between the object and master grating, which leads to a constant phase-shift for every image point. The price to pay is the complication of the experimental set-up, since it is necessary to control accurately the simultaneous variation of two experimental 
parameters. Moreover, phase-shift errors are increased due to the contribution of two error sources.

The application of spatial Fourier transform techniques is also problematic, because it is necessary to introduce a carrier term in the phase, being the spatial frequency of the carrier higher than the characteristic spatial frequencies of the original phase. As it is proposed in [9] a carrier term in the phase can be introduced in Shadow-Moire by tilting the master grating with respect to the average plane defined by the object surface (as Shadow-Moiré is intended to measure flat or almost flat surfaces this "average plane" is unambiguously defined). Due to diffraction (provided that the spatial frequency of the master grating is greater than, approximately, 10 lines $/ \mathrm{mm}$ ), if the distance between master grating and object surface is increased the fringe contrast decreases. Therefore, in the Shadow-Moiré experiment with tilted grating, the fringe contrast varies along the field of view from the maximum value, reached for those points of the object which are closer to the master grating, to a minimum value. Therefore, the value of the tilt angle should be kept small but, since the carrier frequency grows with the tilt angle, the carrier frequency would be also small. Thus, it may happen that, in some experiments, the carrier frequency could not be high enough to use the Fourier transform technique.

A different approach is followed by other methods reported in the literature [10-12]. Xie et al. [10,11] introduce a phase variation by rotating the master grating. In this way, they change the effective pitch, which depends on the rotation angle. Four irradiance images are captured with different rotation angles and the wrapped phase is obtained by applying an arcos function to an algebraic combination of these patterns. Due to the parity of the cosine function the measurement range is limited from 0 to $\pi$ radians. In the work of Arai et al. [12], another solution is found for both static and dynamic problems. In the case of static problems, three different irradiance images corresponding to different phase values are obtained by displacing the master grating vertically (that is, in the direction of the normal of the plane defined by this master grating). The measured irradiance patterns are combined algebraically to get, for a given point of the object, the numerical value of a function $f(z(x, y))$, which depends on the value of the surface height $z(x, y)$ at this point. As $f(z)$ is a well known algebraic function, it is possible to solve numerically the equation $f(z)-\alpha=0$, where $\alpha$ is the numerical value obtained from the three measured irradiance patterns. This process must be applied iteratively to each image point in order to get the surface topography $z(x, y)$. To determine accurately the surface topography it is necessary to know the following experimental parameters: the grating pitch, the distance between camera and light source and the height of the camera over the plane defined by the grating. A similar procedure is applied in the case of dynamic measurements. The main drawback of this method is the high computational cost and processing time required to solve the equation $f(z)-\alpha=0$ for each image point.

To overcome the problem of extraction of quantitative information from a Shadow-Moiré experiment, we propose to apply a direct phase demodulation procedure to get the phase information from a single RGB image. More specifically, we will adapt an asynchronous direct demodulation technique based on the 
calculation of the quadrature sign [14]. This asynchronous direct demodulation algorithm can be employed when the irradiance of the fringe pattern is described by a harmonic function and the sensitivity of the fringe pattern depends monotonically on a given experimental parameter.

If the conditions described in the preceding paragraph are accomplished, the wrapped phase can be directly determined by an arcos operation over the normalized fringe pattern irradiance. As the cosine is an even function the wrapped phase obtained through the arcos operation presents a sign uncertainty. This uncertainty is removed by calculating the sign of the quadrature signal, as it will be shown later.

The application of this algorithm to Shadow-Moiré experiments presents some advantages. First, it keeps a simple experimental set-up, avoiding complications like moving elements and so on. Second, as the phase is obtained from a single image, the method is well suited for the study of dynamic phenomena, such as deformations. We also have developed a procedure to detect the area of interest and to calculate automatically the absolute value of the phase, which is an important feature in Shadow-Moiré.

The paper is organized as follows: in Section 2, we present the theoretical foundations of the method, after this, in Section 3, we give the results of a ShadowMoiré experiment for different objects to evaluate the accuracy and reliability of our technique. We finish with the conclusions and presenting an annex with a brief discussion about the errors associated with the determination of the sign of the quadrature signal from the finite differences of the normalized irradiance patterns, together with the solution proposed to overcome this drawback.

\section{Principle}

\subsection{RGB Shadow-Moiré}

In Fig. 1 we show the experimental Shadow-Moiré set-up employed. It is composed by: (1) a tri-chromatic light source formed by three RGB LEDs, (2) a colour CCD camera and (3) a Ronchi grating placed over the surface to be measured-which will be described by a function $z(x, y)$. In Fig. 1 we can also identify the angles $\alpha_{k}, k=\mathrm{R}, \mathrm{G}, \mathrm{B}$, formed by the illuminating beams with the optical axis of the camera. The peak wavelength of each LED has been selected to match with the maximum spectral sensitivity of the corresponding RGB channel of the CCD camera.

With this experimental set-up we have a polychromatic Shadow-Moire fringe pattern, formed by the superposition of three monochromatic (red, green and blue) patterns with different sensitivity for each one (this is because the light sources are placed in different locations and they illuminate the sample under different angles $\alpha_{k}$ ).

When we take the image of this polychromatic Shadow-Moiré fringe pattern with a colour CCD camera (see Fig. 1), as the peak wavelength of each light source coincides approximately with the maximum spectral sensitivity of each RGB 


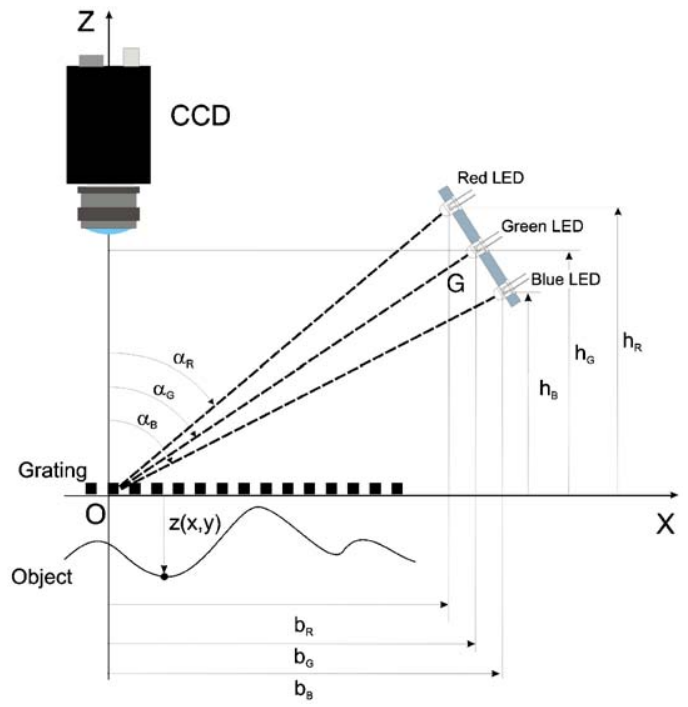

Fig. 1. Scheme of the experimental set-up employed for the RGB Shadow-Moiré.

channel, we capture, with a single RGB snapshot, three monochromatic fringe patterns with different sensitivities. Therefore, the information contained in the polychromatic fringe pattern is demultiplexed so we just need a unique snapshot to extract the wrapped phase using the direct asynchronous demodulation algorithm.

Mathematically, the irradiance at the grating plane $(z=0)$ for each channel of the camera can be described by

$$
I_{k}(x, y)=b_{k}(x, y)+m_{k}(x, y) \cos \left(\Phi_{k}(x, y)\right),
$$

where $b_{k}(x, y)$ is the bias, $m_{k}(x, y)$ is the modulation and $\Phi_{k}(x, y)$ is the phase of the monochromatic Moire fringe pattern formed by the $k$ th source. Supposing that the optical centre of the camera lens and the green source are located, approximately, at equal heights from the plane defined by the Ronchi's grating, then $\Phi_{k}(x, y)$ is described by the following expression:

$$
\Phi_{k}(x, y)=\frac{2 \pi}{p} \frac{b_{k} z(x, y)}{h_{k}+z(x, y)},
$$

where $p$ the grating pitch, $h_{k}$ the height of the $k$ th source from the grating plane and $b_{k}$ the horizontal distance between the optical axis of the CCD camera lens and the $k$ th source (see Fig. 1). In our experiment, we have that the value of $h_{k} \approx 10 \mathrm{~cm}$, while the maximum amount of the absolute value of $z(x, y)$ was, approximately, $0.03 \mathrm{~cm}$. Under these conditions, the following approximation of Eq. (2) holds:

$$
\Phi_{k}(x, y) \approx \frac{2 \pi}{p} \frac{b_{k} z(x, y)}{h_{k}}=\frac{2 \pi}{p} \tan \alpha_{k} z(x, y) .
$$


If we define the sensitivity, for each channel, as $S_{k}=2 \pi \tan \alpha_{k} / p$, we have that $S_{\mathrm{R}}<S_{\mathrm{G}}<S_{\mathrm{B}}$. Therefore, according to Eq. (3) the phase $\Phi_{k}(x, y)$ varies monotonically (in our case, the variation is monotonically decreasing because $z(x, y) \leqslant 0$ for every point) with the sensitivity $S_{k}$. This is the necessary condition that should be accomplished in order to employ the asynchronous direct demodulation algorithm [14].

The first step of this demodulation algorithm is the calculation of the normalized fringe pattern $I_{\mathrm{N}}\left(S_{k}\right)=\cos \left(\Phi\left(S_{k}\right)\right)$ for each channel (the spatial dependence has been removed for simplicity). We have employed the normalization algorithm described in Ref. [13]. The modulus of the wrapped phase $W\{\Phi\}$ can be obtained directly from the normalized fringe pattern by an arcos operation (being $W\{\Phi\}$ the modulo $2 \pi$ phase wrapping operator in the range $[-\pi, \pi])$. For example, for the green channel we have that

$$
\left|W\left\{\Phi\left(S_{\mathrm{G}}\right)\right\}\right|=\arccos \left(I_{\mathrm{N}}\left(S_{\mathrm{G}}\right)\right) .
$$

To determine the sign of $W\{\Phi\}$, the direct asynchronous demodulation algorithm [14] calculates the sign of the quadrature signal $I_{\mathrm{QS}}$, defined as $I_{\mathrm{QS}}\left(S_{k}\right)=-\sin \left(\Phi\left(S_{k}\right)\right)$. As it stated in Ref. [14], the sign of the quadrature signal is related to the sign of the wrapped phase, being $\operatorname{sgn}(W\{\Phi\})=-\operatorname{sgn}\left(I_{\mathrm{QS}}\right)$. According to Ref. [14], provided the phase $\Phi$ presents a monotonic dependence on a given parameter $q$ (in our RGB Shadow-Moire set-up, this parameter is the sensitivity $S_{k}$ ), the sign of the quadrature signal can be calculated as [14]

$$
\operatorname{sgn}\left(I_{\mathrm{QS}}\right)=\operatorname{sgn}\left(\frac{\partial I_{\mathrm{N}}}{\partial q}\right) \operatorname{sgn}\left(\frac{\partial \Phi}{\partial q}\right) .
$$

In our experiment, as the phase $\Phi\left(S_{k}\right)$ is a monotonically decreasing function of the sensitivity, we have that $\operatorname{sgn}\left(\partial \Phi / \partial S_{k}\right)=-1$. Therefore, we just have to calculate the derivative of the normalized intensity $I_{\mathrm{N}}$ with the sensitivity, but as $S_{k}$ is a discrete variable, this derivative should be estimated by finite differences. Therefore, the sign of the quadrature signal is given by the following expression:

$$
\operatorname{sgn}\left(I_{\mathrm{QS}}\right) \approx-\operatorname{sgn}\left(\frac{\Delta I_{\mathrm{N}}\left(S_{\mathrm{G}}\right)}{\Delta S}\right)
$$

where $\Delta S=S_{\mathrm{B}}-S_{\mathrm{G}}$ is the sensitivity difference between the blue and red patterns and $\Delta I_{\mathrm{N}}\left(S_{\mathrm{G}}\right)=I_{\mathrm{N}}\left(S_{\mathrm{B}}\right)-I_{\mathrm{N}}\left(S_{\mathrm{G}}\right)$ the corresponding irradiance difference between these channels. Note that the spatial dependences have been removed for simplicity. Eq. (6) can be simplified considering that $\Delta S>0$, so $\operatorname{sgn}\left(I_{\mathrm{QS}}\right) \approx-\operatorname{sgn}\left(\Delta I_{\mathrm{N}}\left(S_{\mathrm{G}}\right)\right)$. Therefore, the wrapped phase corresponding to the green channel is given by

$$
W\left\{\Phi_{\mathrm{G}}\right\}=\operatorname{sgn}\left(\Delta I_{\mathrm{N}}\left(S_{\mathrm{G}}\right)\right) \arccos \left(I_{\mathrm{N}}\left(S_{\mathrm{G}}\right)\right) .
$$

After unwrapping $W\left\{\Phi_{\mathrm{G}}\right\}$, using a weighted multigrid unwrapping algorithm [15], we can calculate the surface topography $z(x, y)$ using Eq. (3). If we are dealing with noisy fringe patterns, the discrete approximation of the normalized intensity derivative could give rise to errors in the estimation of the proper quadrature signal sign, especially when the values of the phase are close to an integer multiple of $\pi$. 
These errors are due to the asymmetrical location of the red and blue LED with respect to the green one. We show in Appendix A at the end of the paper an analysis of this error source and we give a procedure to correct it.

Finally, we have to point out that, by selecting other discrete derivative operators (we have selected the centred one), only two monochromatic images would have been necessary to calculate properly the phase map. In other words, we could work with only "two colours", but the use of three images ("three colours") reduces the errors in the calculation of the sign of the quadrature signal as it is shown in Appendix A.

\subsection{Automatic detection of the area of interest and zero order fringe calculation}

As it is stated in Ref. [14] the determination of the proper sign of the quadrature signal becomes problematic in low modulation zones of the fringe pattern. To overcome this problem, we have performed an automatic technique for detection of the area of interest by thresholding a modulation map $m(x, y)$ defined as the sum of the modulation maps corresponding to the red, green and blue channels of the CCD camera. The modulation map of each monochromatic pattern is obtained as a result of the normalization algorithm used [13]. Mathematically, the global modulation map is defined as $m(x, y)=m_{\mathrm{R}}(x, y)+m_{\mathrm{G}}(x, y)+m_{\mathrm{B}}(x, y)$ and, the modulation mask is given by

$$
M(x, y)= \begin{cases}1 & \text { if } m(x, y)>m_{\mathrm{th}} \\ 0 & \text { if } m(x, y)<m_{\mathrm{th}}\end{cases}
$$

$m_{\text {th }}$ is the threshold level which has been determined empirically from a number of different experiments (according to these experiments, the selected value for $m_{\text {th }}$ is 1.2).

The only remaining problem is to determine the location of the zero-order fringe in order to set the absolute value of the unwrapped phase. As it is well known, for Shadow-Moire fringe patterns, the zero-order fringe is located just at the contact points between the Ronchi's grating and the test surface. In our experiments, we have worked mainly with flat surfaces with a small indentation placed at their centre. Under these conditions, the contact points between the surface and the grating are located near to the image borders. This means that these contact points and, consequently, the zero-order fringe, are located at low modulation zones of the phase map. But, as these low modulation zones are removed when the modulation mask is applied, it is not possible to determine the location of zero-order fringe. To overcome this problem, we have calculated the edges of the modulation mask $m(x, y)$ using a standard edge detection algorithm. In this way, we obtain a set (denoted by the symbol $\delta \Omega$ ) of points $\left\{x_{i}, y_{i}\right\}$ defining the mask border as a closed curve over the phase map. We have checked in a number of experiments that $\delta \Omega$ is comprised between the zero and the first-order fringes, therefore for the mask edge points we can state that $W\left\{\Phi\left(x_{i}, y_{i}\right)\right\}=\left.\Phi\left(x_{i}, y_{i}\right)\right|_{\forall\left\{x_{i}, y_{i}\right\} \in \partial \Omega}$ (note that subscript $\mathrm{G}$ has been removed for simplicity). Thus, the absolute phase $\Phi_{\text {abs }}$ can be calculated as follows: 
(1) we determine the mean value, $\left.\overline{W[\Phi]}\right|_{\partial \Omega}$, of the wrapped phase $W[\Phi]$ along $\delta \Omega,(2)$ after this, we calculate the mean value of the unwrapped phase, $\left.\bar{\Phi}\right|_{\partial \Omega}$, along the same path $\delta \Omega$, (3) finally, the absolute value of the unwrapped phase $\Phi_{\text {abs }}$ is obtained as

$$
\Phi_{\mathrm{abs}}=\Phi-\left(\left.\bar{\Phi}\right|_{\partial \Omega}-\left.\overline{W[\Phi]}\right|_{\partial \Omega}\right) .
$$

\section{Experimental results}

We have performed an experiment using the set-up drawn in Fig. 1. We employed three LEDs as light sources, with different peak wavelengths: $620 \mathrm{~nm}$ for the red, $530 \mathrm{~nm}$ for the green and $450 \mathrm{~nm}$ for the blue. The CCD camera was a JAI-CV 90 three-chip colour CCD camera and we used a Ronchi grating with 100 lines per inch.
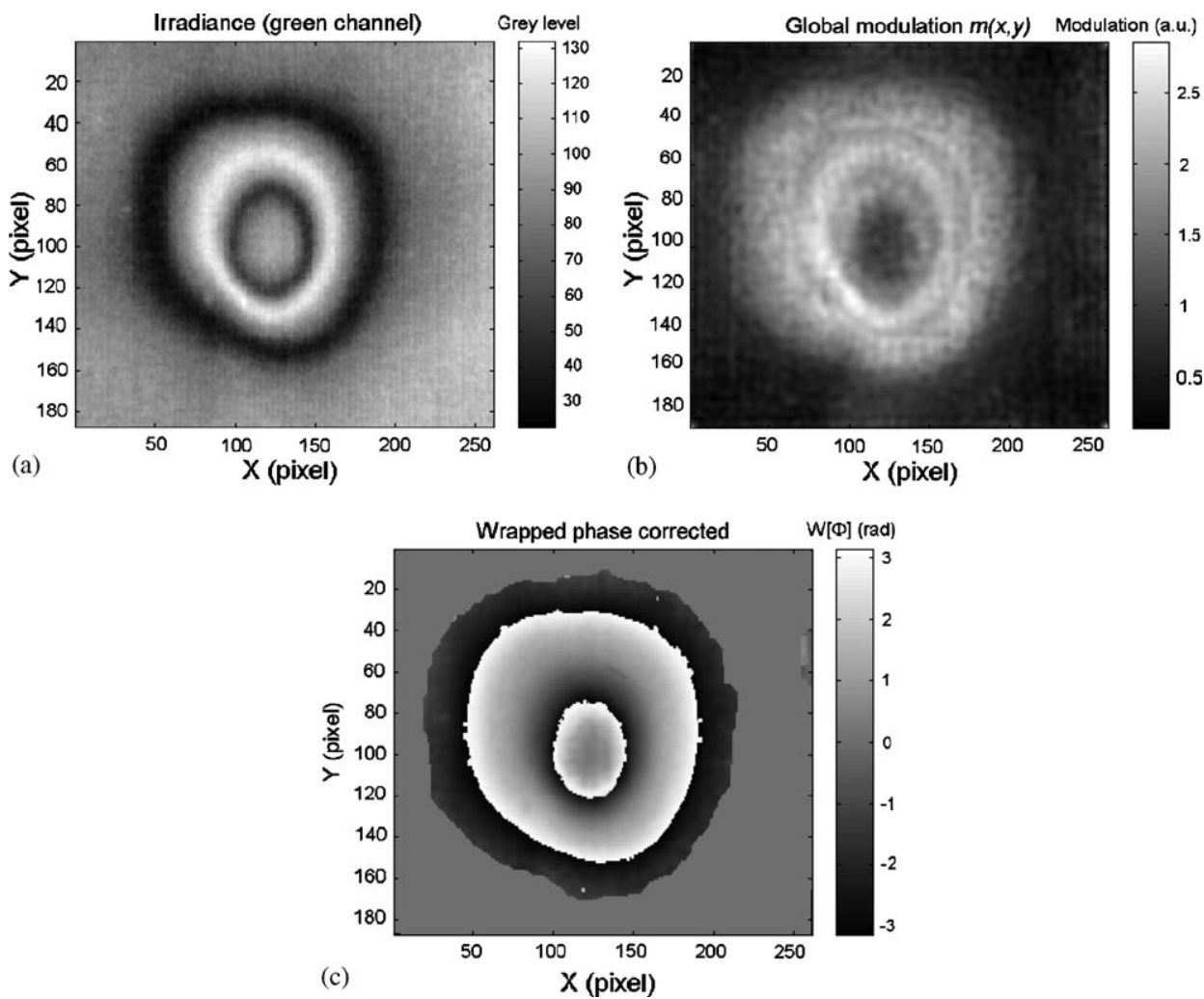

Fig. 2. (a) Irradiance distribution for the Shadow-Moiré pattern produced by an indentation over a flat surface (test object 1) corresponding to the green CCD channel. (b) Global modulation map obtained after applying the normalization algorithm to each channel. It corresponds to the sum of modulations corresponding to red, green and blue irradiance patterns. (c) Wrapped phase map corrected from errors produced by asymmetrical location of red and blue light sources using the procedure of appendix. Note that low modulation zones have been removed using the modulation mask defined from the map depicted in Fig. 2(b). 


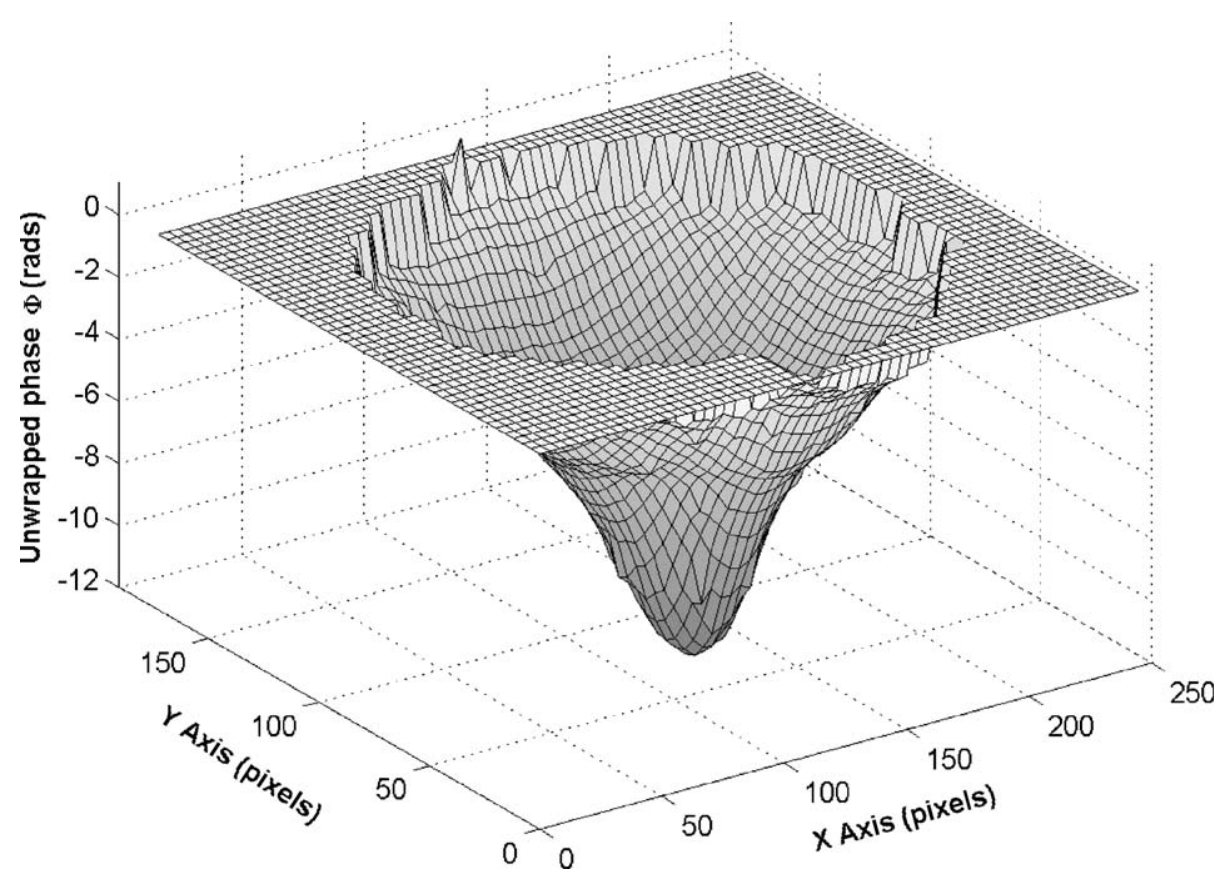

Fig. 3. 3D distribution of the unwrapped phase obtained from the wrapped phase map represented in Fig. 2(c) after phase unwrapping and offset correction as described in text.

We measured three different surfaces. First, we measured the surface of a sheet of composite material employed to form the fuselage of an aircraft. The surface of this sheet was painted in shadow grey and presented a small indentation whose depth was estimated in $400 \mu \mathrm{m}$. The second test object was another fuselage tile painted in flat white. Finally, the third test object was a sheet of fine cardboard with two defects of different concavity over its surface, and it was intended to show the capacity to measure coloured surfaces of arbitrary geometry using our technique.

In Fig. 2, we present the different steps followed to demodulate the phase; Fig. 2(a), shows the irradiance pattern corresponding to the green channel. Fig. 2(b) shows the modulation map $m(x, y)$ obtained after normalizing the irradiance patterns corresponding to the red, green and blue channels of the camera. From this map we have defined the modulation mask. In Fig. 2(c) we have represented the phase map modulo $2 \pi$ with the low modulation zones removed by the modulation map and the errors of the quadrature sign corrected as described before. Finally we have unwrapped the phase using a weighted multigrid technique [14]. The result is shown in Fig. 3 where the $3 \mathrm{D}$ distribution of the unwrapped phase is represented.

Since we can determine the absolute value of the phase using Eq. (11), it is possible to calculate the surface topography from Eq. (7). In Fig. 4(a) we show the irradiance for the green channel corresponding to the second test object. The absolute value of 

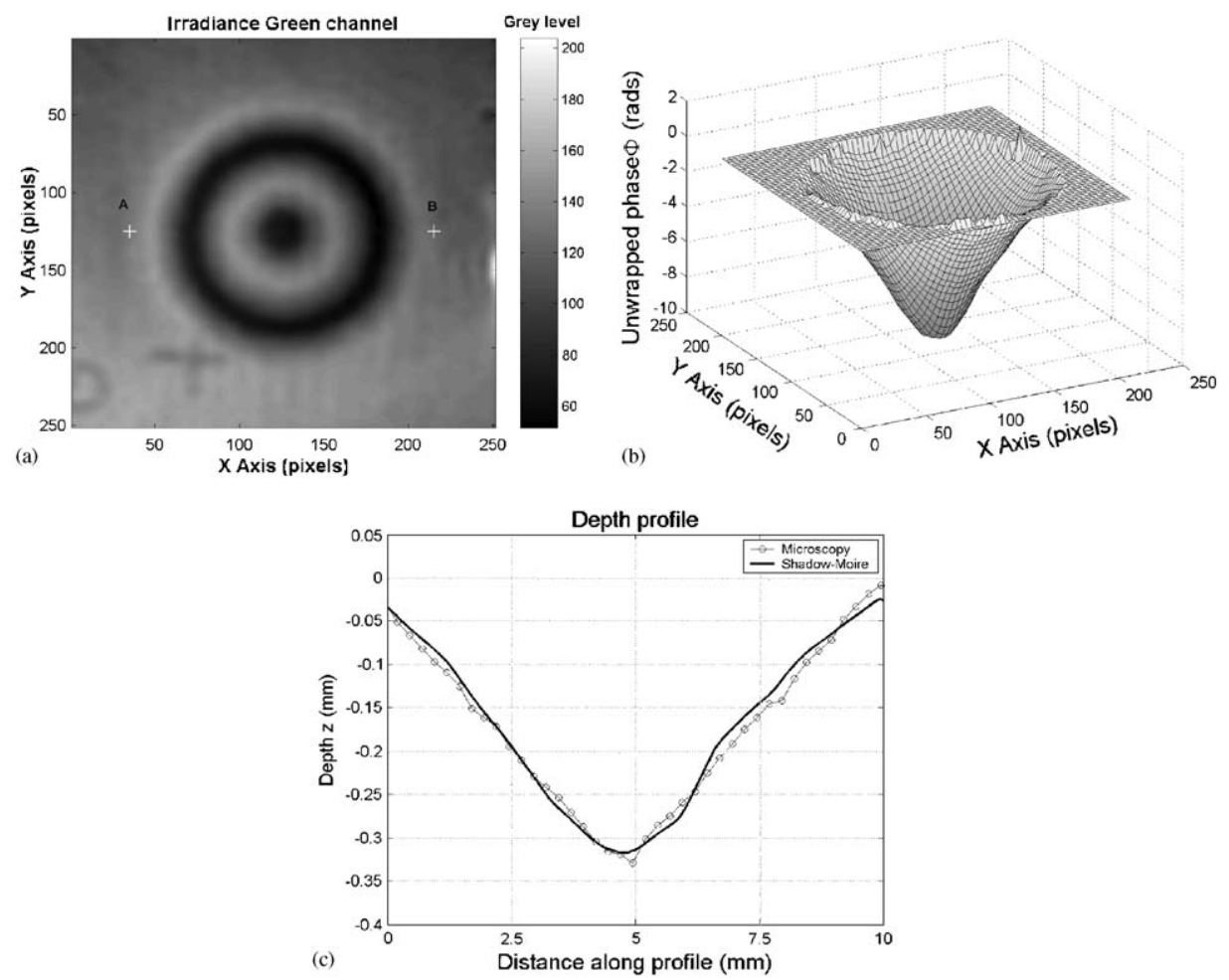

Fig. 4. (a) Irradiance distribution for the Shadow-Moiré pattern produced by an indentation over a flat surface (test object 2) corresponding to the green CCD channel. (b) 3D distribution of the unwrapped phase distribution obtained for the test object 2. (c) Comparison between the depth profile along the line A-B obtained by an alternative optical technique (line with circles) and using our method (solid line) for the second test object.

the unwrapped phase is displayed in Fig. 4b. By inversion of Eq. (7) we have determined the surface topography $z(x, y)$. To test the reliability of the method, we have measured the surface profile located between the points A and B of Fig. 4(a) using a metrology microscope Leica VMM 200 with a lateral resolution of $0.1 \mu \mathrm{m}$ and a depth resolution of $\pm 5 \mu \mathrm{m}$. In Fig. 4(c) we have plotted this profile and we have compared it with the profile, between the same points $\mathrm{A}$ and $\mathrm{B}$, obtained using our method. We can appreciate a good agreement between both curves, showing the ability of our technique to measure the surface topography with a reasonable accuracy and reliability compared to alternative techniques.

Finally, in Fig. 5(a), we show the fringe pattern corresponding to the last test object, a piece of cardboard presenting two defects of different concavity: a protuberance and an indentation. We have selected this object to show that our method is useful to measure the surface topography of non-simple objects with arbitrary geometries, as it can be seen in Fig. 5(b), where we have represented the surface topography of this piece of cardboard. 

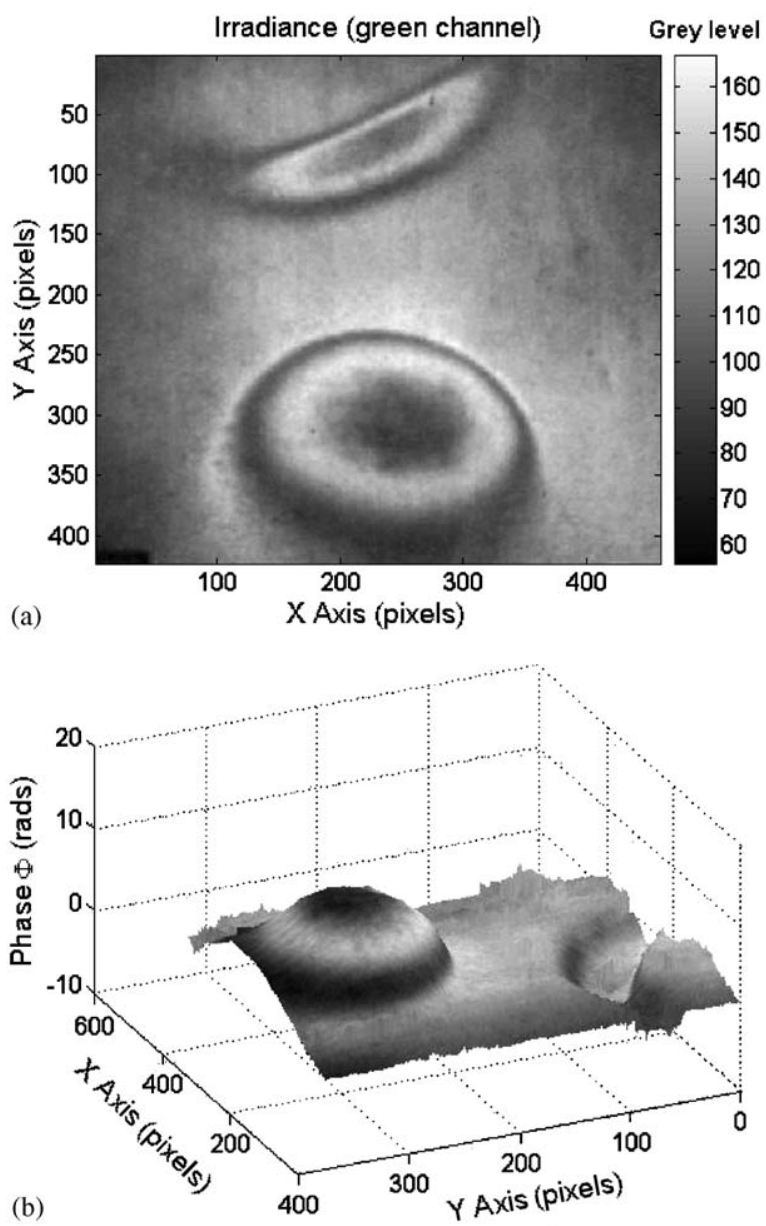

Fig. 5. (a) Shadow-Moire fringe pattern of a cardboard sheet. The surface presents a $x x$ at the low part, and an indentation at the upper part of the fringe pattern. (b) 3D distribution of the unwrapped phase obtained after processing the fringe pattern depicted in Fig. 5(a). The fringe pattern is represented as the texture of the 3D phase distribution surface. Note that our algorithm is able to distinguish between concave and convex surface defects.

\section{Conclusions}

In this work, we present a RGB Shadow-Moiré technique where the phase is demodulated from a single colour image using a direct asynchronous demodulation algorithm while the experimental set-up remains simple (which is one of the general advantages of the Shadow-Moire techniques, simple experimental set-ups). The use of a single exposure to demodulate the phase is an important feature of our technique, because it allows the measurement and control of dynamic phenomena such as deformation, vibrations, etc. 
We have also implemented an automatic method to detect the area of interest of the fringe pattern, together with an automatic procedure to determine the absolute value of the phase, which allows us to calculate exactly the topography of the measured surface. In this way we have a fully automatic RGB Shadow-Moiré technique that can be implemented as a surface inspection tool in different industrial manufacturing and maintenance process.

We have presented a number of experimental results to illustrate the capabilities of our method. We have measured the surface topography of aeronautical components and we have compared the results obtained with alternative optical techniques. We have explored also the suitability of our technique when it is applied to the measurement of irregular surfaces with arbitrary form, finding satisfactory results.

Finally, we have presented an analysis of the errors produced by the asymmetrical location of the light sources together with a method to correct these errors.

\section{Acknowledgements}

This work has been financially supported by the Spanish Ministry of Science and Education; project DPI2002-02104.

\section{Appendix A}

Correction of errors in the determination of phase sign due to asymmetrical location of light sources

As it is stated in Section 2, the sign of the quadrature signal is estimated by a finite difference derivative of the normalized irradiance. This leads to some errors in the estimation of the sign of the quadrature signal errors, which are transmitted to the recovered phase map. Next, we give a general discussion about the quadrature signal sign estimation errors produced by the calculation of the irradiance gradient by finite differences and we propose a method to correct them.

According to Eq. (6) the derivative of the irradiance with respect to the sensitivity $S$ is calculated as

$$
\operatorname{sgn}\left(\frac{\partial I_{\mathrm{N}}}{\partial S}\right) \approx \operatorname{sgn}\left(\frac{\Delta I_{\mathrm{N}}}{\Delta S}\right)=\operatorname{sgn}\left(I_{\mathrm{N}}\left(S_{\mathrm{B}}\right)-I_{\mathrm{N}}\left(S_{\mathrm{R}}\right)\right) .
$$

Substituting $I_{\mathrm{N}}\left(S_{\mathrm{B}}\right)$ and $I_{\mathrm{N}}\left(S_{\mathrm{R}}\right)$ by their explicit expressions, we have that the irradiance difference $\Delta I_{\mathrm{N}}$ can be written as

$$
\Delta I_{\mathrm{N}}=\cos \Phi_{\mathrm{B}}-\cos \Phi_{\mathrm{R}}
$$

If we write the phase variations as $\Phi_{\mathrm{B}}=\Phi_{\mathrm{G}}-\Delta^{-} \Phi$ and $\Phi_{\mathrm{R}}=\Phi_{\mathrm{G}}+\Delta^{+} \Phi$ (the phase is a monotonically decreasing function of the sensitivity, because $z(x, y) \leqslant 0$ over the whole field of view), then, after performing a number of straightforward operations, Eq. (11) becomes

$$
\Delta I_{\mathrm{N}}=2 \sin \left(\Phi_{\mathrm{G}}+\frac{1}{2}\left(\Delta^{+} \Phi-\Delta^{-} \Phi\right)\right) \sin \frac{1}{2}\left(\Delta^{+} \Phi+\Delta^{-} \Phi\right) .
$$


Substituting this expression in Eq. (6) we have that

$$
\begin{aligned}
\operatorname{sgn}\left(I_{\mathrm{QS}}\right) \approx & -\operatorname{sgn}\left\{\sin \left(\Phi_{\mathrm{G}}+\frac{1}{2}\left(\Delta^{+} \Phi-\Delta^{-} \Phi\right)\right)\right\} \\
& \times \operatorname{sgn}\left\{\sin \frac{1}{2}\left(\Delta^{+} \Phi+\Delta^{-} \Phi\right)\right\}
\end{aligned}
$$

According to this equation, when $\Delta^{+} \Phi \neq \Delta^{-} \Phi$ there appears a shift between $\Delta I_{\mathrm{N}}$ and $\sin \Phi_{\mathrm{G}}$. This shift produces errors in the estimation of the sign of the quadrature signal using Eq. (9), especially for those points of the fringe pattern for which the value of $\Phi_{\mathrm{G}}$ is near to $\pi$. This problem is reduced if $\Delta^{+} \Phi \ll$ and $\Delta^{-} \Phi \ll$ (which implies that, in our experiment, the locations of the red and blue light sources should be close to that of the green one), but, in this case, $\Delta I_{\mathrm{N}}$ will also be small due to the modulating term $\sin \frac{1}{2}\left(\Delta^{+} \Phi+\Delta^{-} \Phi\right)$, and the estimation of the sign will be perturbed even by a small amount of noise.

This problem can be corrected as follows: if we suppose that $\left|\Delta^{+} \Phi+\Delta^{-} \Phi\right|<2 \pi$, the sign of the quadrature signal is estimated as $\operatorname{sgn}\left(I_{\mathrm{QS}}\right) \approx-\operatorname{sgn}\left\{\sin \left(\Phi_{\mathrm{G}}+\Delta \Phi\right)\right\}$, where $\Delta \Phi=\frac{1}{2}\left(\Delta^{+} \Phi-\Delta^{-} \Phi\right)$. Let us denote by $\mathrm{QS}_{\Delta}$ to this estimation of the sign of the quadrature signal.

From the properties of the sine function, if $\Delta \Phi>0$ the relation of $\mathrm{QS}_{\Delta}$ with the true value of the sign of the quadrature signal (denoted by $\mathrm{QS}=\operatorname{sgn}\left(I_{\mathrm{QS}}\right) \equiv$ $\left.-\operatorname{sgn}\left(\sin \left(\Phi_{\mathrm{G}}\right)\right)\right)$ is

$$
\mathrm{QS}\{I\}_{\Delta}=\left\{\begin{array}{c}
-\mathrm{QS}\{I\}, \Phi \in[2 n \pi, \Delta \Phi+2 n \pi] \\
\mathrm{QS}\{I\}, \Phi \in[\Delta \Phi+2 n \pi,(2 n+1) \pi], \\
-\mathrm{QS}\{I\}, \Phi \in[(2 n+1) \pi,(2 n+1) \pi+\Delta \Phi] \\
\operatorname{QS}\{I\}, \Phi \in[(2 n+1) \pi+\Delta \Phi,(2 n+2) \pi],
\end{array}\right.
$$

where $n$ is an integer number. When $\Delta \Phi<0$, we have to change $\Delta \Phi$ by $\Delta \Phi+\pi$ in Eq. (14). Thus if we denote by $W\{\Phi\}_{\Delta}$ the phase map recovered by Eq. (9) using $\mathrm{QS}_{\Delta}$, we will obtain sign errors in $W\{\Phi\}_{\Delta}$ around the phase values $\Phi=n \cdot \pi$.

If the phase is monotonic with phase variations high enough in relation to the noise level, the spurious phase jumps can be filtered by simply thresholding the phase map and then interpolating the phase inside the thresholded region. In the case of the phase errors located at the zero crossing of the wrapped phase the regions to interpolate can be located by looking for the points for which $|W\{\Phi\}|<\varepsilon$, being $\varepsilon$ the selected threshold. In the case of the errors located at the $2 \pi$ phase jumps the regions to interpolate can be easily located by selecting the points for which $|W\{W\{\Phi\}+\pi\}|<\varepsilon$.

In consequence, providing that the locations of the three light sources guarantee that the condition $\left|\Delta^{+} \Phi+\Delta^{-} \Phi\right|<2 \pi$ holds, then it is possible to correct the errors discussed above, making possible the asynchronous demodulation even if $\Delta^{+} \Phi \neq \Delta^{-} \Phi$. This is important, because, in our case, the only way to get the amount of $\Delta^{+} \Phi$ equal to that of $\Delta^{-} \Phi$ is to locate the red and blue sources completely symmetrical with respect to the illumination direction of the green one defined by the line OG of Fig. 1, but doing this would require a very accurate placement of the red 
and blue sources (small position tolerances) which could be difficult to achieve and could also increase the complexity of the experimental set-up.

\section{References}

[1] Patorski K. Handbook of the Moiré fringe technique. New York: Elsevier; 1993.

[2] Robinson D, Reid G. Interferogram analysis. Bristol: Institute of Physics Publishing; 1993.

[3] Malacara D, editor. Optical shop testing. New York: Wiley Interscience; 1992.

[4] Kreiss T. Holographic interferometry. Berlin: Akademie Verlag Series in Optical Metrology; 1996.

[5] Mavoisin G, Brémard F, Lagarde A. Three-dimensional phase reconstruction by phase-shifting Shadow Moiré. Appl Opt 1994;33:2163-9.

[6] Ladak H, Decraemer W, Dirckx J, Funnell WR. Systematic errors in small deformations measured by use of Shadow-Moiré topography. Appl Opt 2000;39:3266-74.

[7] Shapira I, Voloshin A. Fractional Moiré fringe analysis by optimization. Opt Eng 1992;31:838-45.

[8] D'Acquisto L, Fratini L, Siddiolo AM. A modified Moiré technique for three-dimensional surface topography. Meas Sci Tech 2002;13:613-22.

[9] Yoshiwaza T, Tomisawa T. Shadow Moiré topography by means of the phase-shift method. Opt Eng 1993;32:1668-74.

[10] Xie X, Atkinson J, Lalor M, Burton D. Three-map absolute Moiré contouring. Appl Opt 1996;35:6990-5.

[11] Xie X, Lalor M, Burton D, Shaw M. Four-map absolute distance contouring. Opt Eng 1997;36: $2517-20$.

[12] Arai Y, Yokozeki S, Yamada T. Fringe-scanning method using a general function for Shadow Moiré. Appl Opt 1995;34:4877-82.

[13] Quiroga JA, Gómez-Pedrero JA, García-Botella A. Algorithm for fringe pattern normalization. Opt Commun 2001;197:43-51.

[14] Quiroga JA, Gómez-Pedrero, Terrón-López MJ, Servin M. Temporal demodulation of fringe patterns with sensitivity change. Opt Commun 2005;253:266-75.

[15] Ghiglia D, Pritt M. Two-dimensional phase unwrapping: theory, algorithms, and software. New York: Wiley; 1998. 